\title{
THE STUDY OF RESIDENTIAL AREAS EXTRACTION BASED ON GF-3 TEXTURE IMAGE SEGMENTATION
}

\author{
SHAO Guangzhou ${ }^{1 *}$, LUO Heng $^{1}, \quad$ TAO Xiaodong ${ }^{2}, \quad$ LING Ziyan ${ }^{1,2}$, HUANG Yu ${ }^{1,2}$ \\ ${ }^{1}$. Geomatics Center of Guangxi Zhuang Autonomous Region, Nanning 530023, China - (271283876, 182872242)@qq.com \\ 2. The Academy of National Geographical Condition of Guangxi Zhuang Autonomous Region, Nanning 530023, China -
}

(417420925, 352289018, 23993891)@qq.com

KEY WORDS: Residential Areas Extraction, GF-3, Backscattering Coefficient, Image Filtering, Texture Feature, Image Segmentation

\begin{abstract}
:
The study chooses the standard stripe and dual polarization SAR images of GF-3 as the basic data. Residential areas extraction processes and methods based upon GF-3 images texture segmentation are compared and analyzed. GF-3 images processes include radiometric calibration, complex data conversion, multi-look processing, images filtering, and then conducting suitability analysis for different images filtering methods, the filtering result show that the filtering method of Kuan is efficient for extracting residential areas, then, we calculated and analyzed the texture feature vectors using the GLCM (the Gary Level Co-occurrence Matrix), texture feature vectors include the moving window size, step size and angle, the result show that: window size is $11 * 11$, step is 1 , and angle is $0^{\circ}$, which is effective and optimal for the residential areas extracting. And with the FNEA (Fractal Net Evolution Approach), we segmented the GLCM texture images, and extracted the residential areas by threshold setting. The result of residential areas extraction verified and assessed by confusion matrix. Overall accuracy is 0.897 , kappa is 0.881 , and then we extracted the residential areas by SVM classification based on GF-3 images, the overall accuracy is less 0.09 than the accuracy of extraction method based on GF-3 Texture Image Segmentation. We reached the conclusion that, residential areas extraction based on GF-3 SAR texture image multi-scale segmentation is simple and highly accurate. although, it is difficult to obtain multi-spectrum remote sensing image in southern China, in cloudy and rainy weather throughout the year, this paper has certain reference significance.
\end{abstract}

\section{INTRODUCTION}

GF-3 satellite, the first $\mathrm{C}$ band and multi-polarization SAR satellite in China, is the Synthetic Aperture Radar (SAR) satellite mission with scientific and commercial applications, which was launched in August 2016. GF-3 satellite is the important part of China high-resolution earth observation system with the abilities of high spatial resolution, high temporal resolution, high spectral resolution and high precision, GF-3 satellite can provide reliable and stable high-resolution SAR images, and can frequently provide SAR images with resolution of 1 to 500 meters in different application modes. As a large amount of GF-3 data are available for resident area analysis, witch result in an urgent demand on GF-3 image interpretation. the residential areas, as an important activity place for human beings, timely monitoring of changes in residential areas, play an important role in protecting land resources and environment.

At present, extraction of residential information for GF-3 images is relatively rare. But there are several methods for extracting residential area information from other highresolution SAR images, The main methods are the classification and extraction based on support vector machines(SVM), the method based on supervised classification of SAR texture images, and extracting residential area based on the combination of the digital number value and texture.

This paper presents a method of high-efficiency and highprecision extraction of residential areas process. Firstly we calculated the Gray Level Co-occurrence Matrix (GLCM) of

\footnotetext{
* Corresponding author

E-mail address: 271283876@qq.com (SHAO Guangzhou).
}

GF-3 satellite images, and segmented the GLCM texture images through multi-scale segmentation of fractal network evolutionary algorithm, and then we extracted the residential areas based on the image objects.

\section{DATA AND STUDY AREA}

The study area is Xiangzhou County, which belongs to Laibin City, Guangxi Zhuang Autonomous Region, located between longitude $109^{\circ} 25^{\prime}-110^{\circ} 06^{\prime} \mathrm{E}$ and north latitude $23^{\circ} 44^{\prime}-24^{\circ} 18^{\prime}$, the study area is both urban and rural, with mountainous, alpine, and hilly terrain. These conditions are suitable for this study. The study chooses the standard stripe and dual polarization SAR images of GF-3 as the basic data. The resolution of GF-3 image is 8 meters, the shooting time is April 30, 2017, and the incidence angle is 30.74 degrees.

\section{GF-3 IMAGES PREVIOUS PROCESS}

The GF-3 Image data we received is the slope single look complex data (L1 level), it's the standard stripe and dual polarization SAR images data. We need to preprocess the data to L4 industry application products, the GF-3 slope single look complex data (SLC) image preprocessing workflow include radiometric correction, amplitude data conversion, multi-look image conversion, image filtering and geocoding. 


\subsection{Radiometric correction}

The radar sensor measures the ratio of the transmitted pulse to the received signal strength, the ratio is backscatter, backscattered intensity information after radiometric calibration is not affected by geometric errors in SAR data observations, for example different SAR sensors or different reception modes. Data can be compared and analyzed due to normalization to the same standard, there are radiation errors in single look complex data, which is affected by several kinds of disturbances coming from error sources. So we need to calibrate the radiation and convert the input signal to radar backscatter coefficient, In the study, we used the radiation correction module of PIE (Pixel Information Expert) for radiation correction.

\subsection{Amplitude data conversion and multi-look conversion}

The amplitude characteristic is one of the most important features of SAR data. Based on the SAR amplitude image data, the target information of the object can be extracted. Therefore, the SAR single look complex data (SLC) should be converted into SAR amplitude data.

Since the GF-3 single look complex data (SLC) is a coherent superposition of radar echo signals scattered from a single pixel, there is a lot of noise in the intensity information. So we convert the SLC data into multi-look image data, which can improve the visual effect of the image and improve the estimation accuracy of backscattering for each pixel, which is the average of the azimuth and distance of the GF-3 SLC data. The result is the multi-look intensity data. The data with multi-look conversion reduces spatial resolution and improves data radiation resolution or the intensity information.

The multilook number is calculated from the distance and azimuth resolution of the slant range, and the incident angle. the ground resolution of the standard stripe and dual polarization SAR image of GF-3 is 8 meters. In order to obtain the same ground resolution for the azimuth and distance directions, the azimuth multilook number is set to 3 and the distance multilook number is set to 3 by calculation.

\subsection{Image filtering}

According to the characteristics of coherent noise in GF-3 images, we compared and analyzed various popular filtering methods, such as mean filtering, Frost filtering, enhanced Lee filtering, Lee filtering, median filtering, etc. The quality of images filtered by various filtering methods was evaluated using the mean, standard deviation, smoothness index, PM value, information entropy, and average gradient. And compared and analyzed the result of noise removing, we choose a filtering method that has both strong ability to suppress noise and better preservation of Texture Information from GF-3 Images. the evaluation results show that, the result of using enFrost filtering method is the smoothest effect, and the result of using Kuan filtering is the roughest effect, the results are reported in Table 1. the Kuan filtering is most beneficial to the extraction of residents area (see Figure 1).

\begin{tabular}{|c|c|c|c|c|c|}
\hline & mean & $\begin{array}{l}\text { standard } \\
\text { deviation }\end{array}$ & $\begin{array}{l}\text { PM } \\
\text { value }\end{array}$ & $\begin{array}{l}\text { smoothness } \\
\text { index }\end{array}$ & $\begin{array}{l}\text { information } \\
\text { entropy }\end{array}$ \\
\hline Kuan & 0.1786 & 0.274196 & 1.00607 & 0.651647 & 432.5336 \\
\hline Frost & 0.1774 & 0.217844 & 0.99922 & 0.814633 & 457.6113 \\
\hline enFros & 0.177 & 0.217206 & 0.9999 & 0.817629 & 467.1894 \\
\hline enLee & 0.1776 & 0.222567 & 1.0000 & 0.798034 & 461.3620 \\
\hline
\end{tabular}

Table 1. Evaluation index of various filtering methods

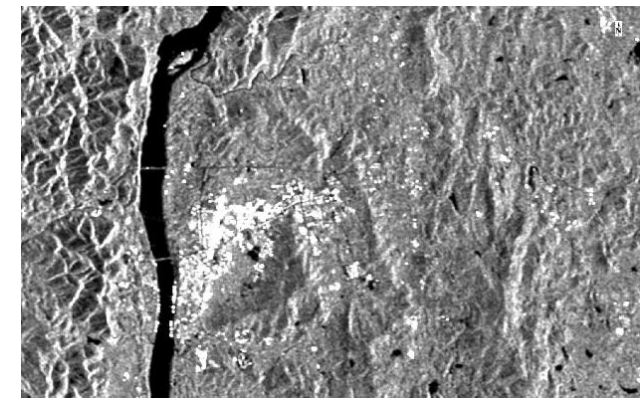

Figure 1. The Kuan filtered GF-3 SAR image

\subsection{Image geocoding}

The original image is encoded into the radar coordinate system and is a slant range coordinate system. In practical applications, the GF-3 SAR data needs to be transferred from the slant range coordinate system to the geographic coordinate system, the conversion process is geocoding, the commonly geocoding methods include geocoding ellipsoid correction(GEC) and geocoding of Terrain Correction(GTC), in this study, we used geocoding ellipsoid correction method for geocoding [3], with the image geocoding module of PIE (Pixel Information Expert). The results of geocoding ellipsoid correction GF-3 SAR images in the study area are shown in Figure 2.

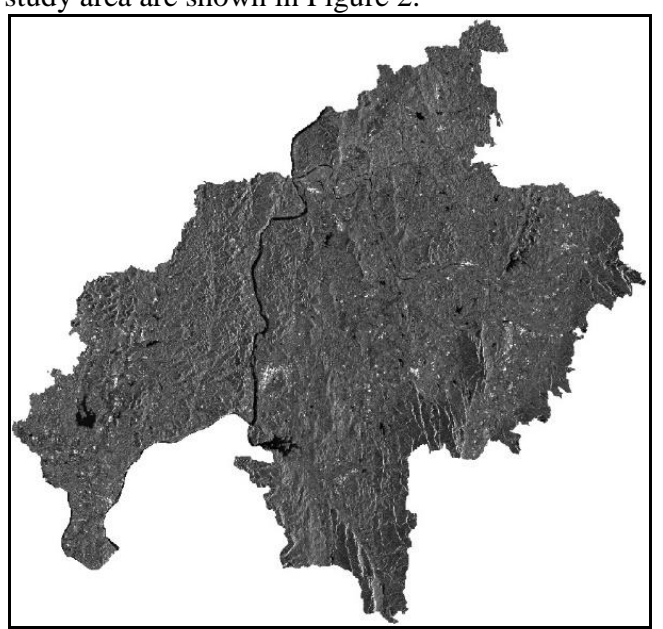

Figure 2. The geocoded GF-3 SAR image

\section{MULTI-SCALE SEGMENTATION OF GF-3 TEXTURE IMAGE}

\subsection{Selection of texture features}

The factors affecting the target radar echo intensity and modes in residential areas mainly include radar system parameters, land surface properties and geographical environmental variables. Radar system parameters include wavelength, polarization, angle of incidence, direction of view, etc. and surface properties include geographical features and dielectric properties. Geographical environmental variables include landform type, vegetation Coverage, climate, etc. Residential areas include residential buildings, roads, public facilities, landscaping, and production facilities ${ }^{[4][5]}$. The characteristics of the residential area on the GF-3 SAR image are the result of the comprehensive response of various factors. With comparing 
and analyzing a large number of GF-3 SAR data, the residents area generally display higher overall signal intensity than the surrounding areas on the GF-3 SAR image, however, the residential area contains a number of discrete, small areas of darkness, and the building groups are arranged in the same direction, this makes the residential areas have better textures on GF-3 SAR images. At present, the typical methods for analyzing the texture of GF-3 SAR images include the gray level co-occurrence matrix, fractal theory, Markov model and so on. In this study, we used GF-3 SAR images to generate gray level co-occurrence matrix texture images, performed multiscale segmentation on texture images, and extracted residential areas based on segmentation results. we contrasted the Energy , Entropy, Contrast, Correlation, variance, Mean, and Homogeneity of the gray level co-occurrence matrix, we use homogeneous texture features for multi-scale segmentation to extract residents area, homogeneity measures the value of the local variation of the SAR image texture, and a large value indicates that there is a lack of change between different regions of the image texture, and the locality is very uniform.

The calculation parameters of the gray-level co-occurrence matrix include the calculation moving window size, calculation moving step size and moving direction, we calculated and selected the gray level co-occurrence matrix parameters, in order to get the best gray-level co-occurrence matrix results. The smaller moving window size is likely to cause the loss of GF-3 SAR texture information, but too large moving window size will result in the loss of GF-3 SAR image edge information. We have experimentally analyzed that SAR texture images with a moving window size of 7 exhibit too much detail, when the window size is 13 , the edges of the SAR texture image of the residential area also appear blurred, and the larger the moving window size, the more blurred the SAR texture image. When the moving window size is 11 , the step size is 1 , and the direction is 0 , the SAR texture image is not complicated, and the edge information is also kept well. Therefore, the moving window size is $11^{*} 11$, the step size is 1 , and the direction is 0 is the most appropriate. The homogeneity result of the study area is shown in Figure 3 to Figure 4.

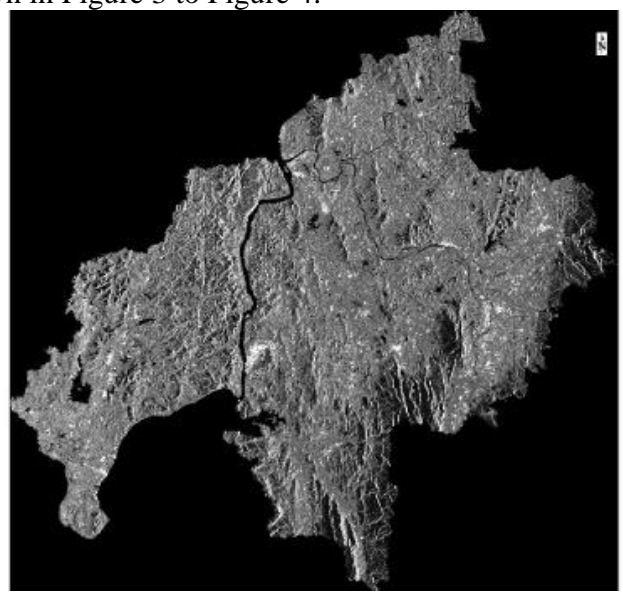

Figure 3. The homogeneity of the gray-level co-occurrence matrix

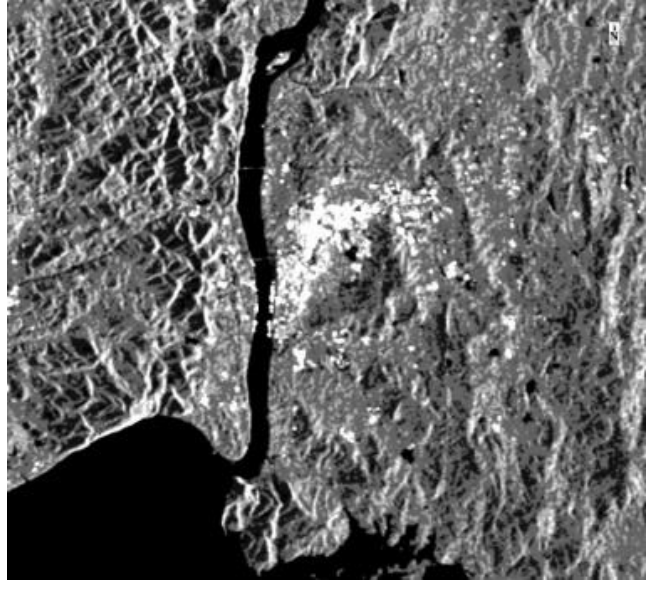

Figure 4. Enlarged view of the homogeneity result

\subsection{Multi-scale segmentation of GF-3 texture image}

The multi-scale segmentation of SAR images can use the least heterogeneous region merging algorithm. The basic thinking is: The merging of pixels in image segmentation begins with any pixel in the image, and first merges individual pixels into smaller image objects. The smaller image objects are merged into larger polygon objects. During the segmentation process, the polygon objects grow continuously. However, the internal heterogeneity must be minimized to achieve the entire image with a given segmentation threshold. The average heterogeneity of the objects is the smallest [8]. The necessary precondition for high-precision extraction of residential areas is appropriate multi-scale segmentation, and the segmentation results directly affect the accuracy of residential extraction [9].

The most representative region merge algorithm with the smallest heterogeneity is the FNEA (Fractal Net Evolution Approach). The key of this algorithm is to determine the heterogeneity between two image objects [10]. For a given feature space, when two image objects are close together in the feature space, they are considered to be similar or homogeneous. The heterogeneity of image objects in the Fractal Net Evolution Approach consists of two parts: color and shape. we derive the acquisition $\mathrm{f}$ (heterogeneity) through:

$$
f=w_{\text {color }} \times h_{\text {color }}+w_{\text {shape }} \times h_{\text {shape }}
$$

Where $h_{\text {color }}$ is the color heterogeneity of the image object, $h_{\text {shape }}$ is the shape heterogeneity of the image object, $w_{\text {color }}$ is the weight of color and $w_{\text {shape }}$ is the weight of shape. Shape heterogeneity is again composed of two parts: compactness and smoothness:

$$
h_{\text {shape }}=w_{\text {cmpct }} h_{\text {cmpct }}+w_{\text {smooth }} h_{\text {smooth }}
$$

Where $h_{\text {cmpct }}$ is the compactness of the image object , $h_{\text {smooth }}$ is the smoothness of the image object, $w_{\text {cmpct }}$ is the weight of compactness and $w_{\text {smooth }}$ is the weight of smoothness. 
This study was validated by a large number of experiments and the extraction results of residents, and the best segmentation scale parameters that are most suitable for GF-3 SAR image are shown in Table 2. The results of Multi-scale segmentation are shown in Figure 5.

\begin{tabular}{|c|c|c|c|c|}
\hline segmentation scale & $\mathrm{W}_{\text {color }}$ & $\mathrm{W}_{\text {shape }}$ & $\mathrm{W}_{\text {cmpct }}$ & $\mathrm{W}_{\text {smooth }}$ \\
\hline 20 & 0.8 & 0.2 & 0.5 & 0.5 \\
\hline
\end{tabular}

Table 2. Multi-scale segmentation parameters of GF-3 SAR image

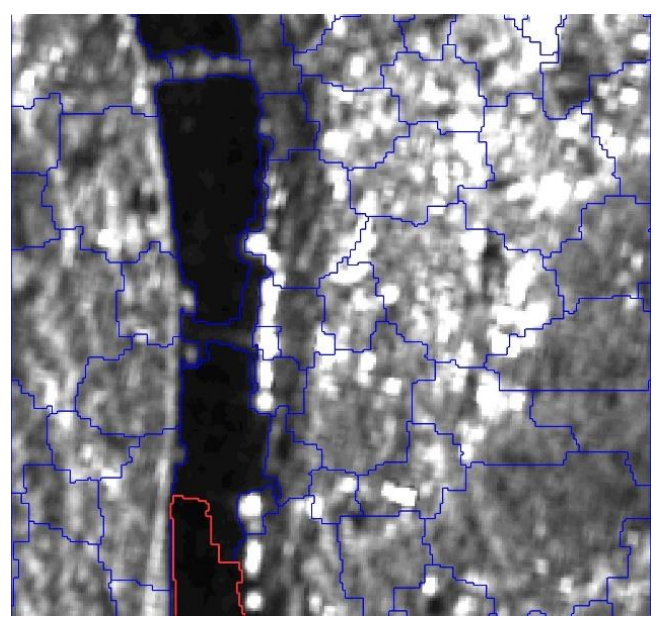

Figure 5. The results of Multi-scale segmentation of GF-3 SAR image

\section{RESIDENTIAL AREA EXTRACTION}

By setting a threshold value, the image objects of GF-3 SAR image multi-scale segmented is classified into three categories: residential area, water bodies, and non-resident area. The homogenous image object has a value greater than 0.29 is classified as a residential area. A homogeneity image object less than 0.165 is classified as Water bodies, homogeneity image objects greater than 0.165 and less than 0.29 are classified as non-residential (see Figure 6).

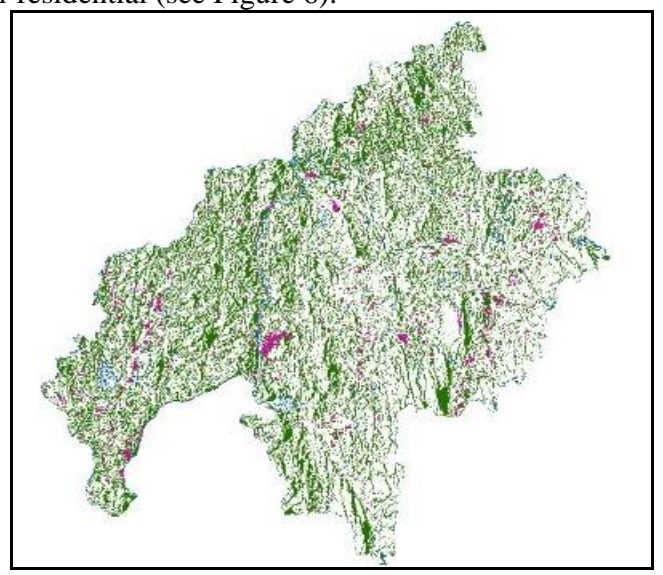

Figure 6 . The results of residential area extraction

\section{ACCURACY EVALUATION AND COMPARISON}

\subsection{Classification accuracy evaluation}

By randomly generating 213 sample points within the study area, and using April 2017 high-resolution multi-spectral images, three-dimensional street views, and other land classification thematic maps as reference materials in the same period, experienced professionals interpreted and classified the samples. The accuracy of the evaluation is based on the result of the samples classification. The distribution of random points is shown in Figure 7.

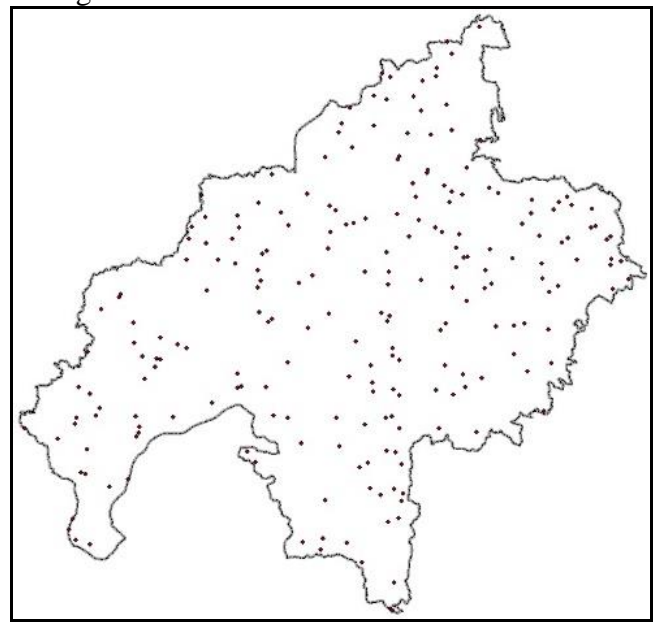

Figure 7. Distribution of random samples

We evaluated the accuracy by calculating the confusion matrix, the producer accuracy of residential area extraction by calculating the confusion matrix is 0.875 , the producer accuracy of water bodies extraction by calculating the confusion matrix is 0.864 , the producer accuracy of non-resident area extraction is 0.901 , overall accuracy by calculating the confusion matrix is 0.897 , and Kappa coefficient is 0.651 . The confusion matrix is shown in Table 3.

\begin{tabular}{|ccccc|}
\hline $\begin{array}{c}\text { User Class } \\
\text { Sample }\end{array}$ & $\begin{array}{c}\text { residential } \\
\text { area }\end{array}$ & water bodies & $\begin{array}{c}\text { Other land } \\
\text { cover }\end{array}$ & Sum \\
\hline $\begin{array}{c}\text { residential } \\
\text { area }\end{array}$ & 7 & 0 & 1 & 8 \\
water bodies & 0 & 19 & 3 & 22 \\
$\begin{array}{c}\text { Other land } \\
\text { cover }\end{array}$ & 13 & 6 & 174 & 193 \\
Sum & 20 & 25 & 178 & 0 \\
\hline
\end{tabular}

Table 3. The confusion matrix

\subsection{Comparison}

We compared this study method with SVM classification method, which is based on image pixel for extracting residential area. Using the pixel-based SVM classification, the producer accuracy of the residential area extraction is 0.805 , the producer accuracy of the water body is 0.856 , the producer accuracy of other land cover is 0.801 , the overall accuracy is 0.807 , and the Kappa coefficient is 0.621 . (The residential area extraction based on SVM classification shown in Figure 8). The extraction accuracy of residential areas based on pixel-based SVM 
classification is lower than that based on multi-scale segmentation of GF-3 SAR texture images, and many broken and small maps are misclassified for residential areas and do not belong to residents. The reasons for the above misclassification include: for high-resolution SAR images, pixel-based SVM image classification cannot fully utilize the rich information in high-resolution SAR data, cannot overcome the impact of inherent "speckle" noise on classification and cannot extract objects of interest, etc. [12], SAR image classification based on multi-scale segmentation divide the image into object, and extracting information by image object can effectively solve the effects of SAR image's scale effect and noise suppression.

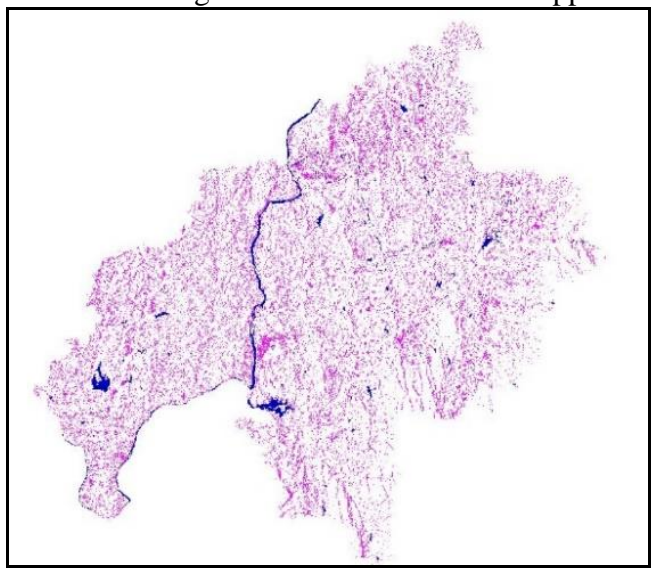

Figure 8. Residential area extraction based on SVM classification

\section{CONCLUSIONS}

In this paper, the GF-3 SAR is used for the extraction of residential areas, and the GF-3 SAR is produced and discussed from grade 0 to grade 3 products. Firstly, we calculated the gray level co-occurrence matrix of GF-3 SAR image, and multi-scale segmented the texture images using fractal net evolution approach, set a certain threshold as a criterion for determining whether an image object is a residential area. After doing a lot of experiments, we obtained filtering methods and multi-scale segmentation parameters, which is suitable for residential area extraction, classification based on pixel-based SVM and based on multi-scale segmentation of texture features and set a certain threshold for residential areas extraction, we reached the conclusion that, residential areas extraction based on GF-3 SAR texture image multi-scale segmentation is simple and highly accurate. The influencing factors of this method include: complex reflection characteristics of residential areas in GF-3 SAR image, multi-scale segmentation parameters, and threshold selection. At the same time, the segmentation scale must be determined through multiple trials. The residential area extraction process takes a long time and requires professional knowledge and well experienced. However, it is difficult to obtain multi-spectrum remote sensing image in southern China, in cloudy and rainy weather throughout the year. Studying the areas in resident area extraction and environmental monitoring based on the GF-3 SAR image has practical application value. This paper has certain reference significance.

\section{REFERENCES}

Yue Y, Gong J,Wang D. The extraction of water information based on SPOT5 image using object-orientedmethod. 18 th International Conference on Geoinformatics, 2010, 18-20 June 2010; 2010. p. 1-5.
CHEN Er-xue ,LI Zeng-yuan(Institute of Forest Resources Information Techniques, State Laboratory for Forest Remote Sensing and Information Techniques, Chinese Academy of Forestry, Beijng 100091);Method of Geocoding ALOS PALSAR Image to the Earth Ellipsoid[J];Remote Sensing Information;2008-01.

Chen Q H, Liu X G, Chen Q. Woodland and building extraction from polarimetric SAR images by integrated multi-features[J]. Science of Surveying \& Mapping, 2014.

Crosetto, M., 2002. Calibration and validation of SAR interferometry for DEM generation. ISPRS Journal of Photogrammetry and Remote Sensing 57, 213-227.

Fan, W. U., Chao, W., \& Hong, Z. ,2005. Residential areas extraction in high resolution sar image based on texture features. Remote Sensing Technology \& Application.

Huang, G., Yang, S., Wang, N., \& Zhang, J. ,2013. Block combined geocoding of airborne insar with sparse gcps. Acta Geodaetica Et Cartographica Sinica.

Qian, Q. J., Rui, X., Lei, Z., Yan, C. Z., \& Bing-Fang, W. U. ,2005. Land cover extraction based on object oriented analysis. Remote Sensing Technology \& Application.

Tsutsumida, N., Comber, A., 2015. Measures of spatiotemporal accuracy for time series land cover data. Int. J. Appl. Earth Obs. Geoinf. 41, 46-55.

Zhang, Q. System Design and Key Technologies of the GF-3 Satellite. Acta Geodaetica et Cartographica Sinica 2017, 46, 269-277. 